\title{
Frequency Plan Optimization Based on Genetic Algorithms for Cellular Networks
}

\author{
Hicham Megnafi
}

\begin{abstract}
Cellular networks are constantly evolving to ensure a better Quality of Service (QoS) and quality of coverage ever more important. The radio cellular systems are based on frequency allocation. In this context, frequency allocation principle consists in choosing an optimal frequency plan to meet traffic demand constraints and communication quality while minimizing the radio interferences. This paper proposes an optimal frequency allocation approach based on genetic algorithms to minimize co-channel and adjacent channel interference. The validation of this new approach is confirmed by the results of the work we have done in the GSM network. In fact, we used the file obtained by the OMC-R, which defines the adjacent cells of each cell and the frequencies allocated to the considered area. The results obtained clearly show the effectiveness and robustness of the approach used.
\end{abstract}

Index Terms - cellular networks, radio optimization, frequency allocation, radio interference, genetic algorithms, drive test.

\section{INTRODUCTION}

Network planning and optimization as well as customer satisfaction are the most critical aspects of the mobile operator activity [1]. One of the main goals of any mobile network operator is a well-planned radio access network, for that, operators put a lot of effort to improve their radio networks [2]. The proposed research work is based on planning and optimization of the frequency plan in the Global System for Mobile communication (GSM).

Currently, several applications are developed through the use of the GSM network, such as Machine to Machine (M2M) which use the Extended Coverage GSM for Internet of Things standard (EC-GSM-IoT) [3]. For example, the pilot EC-GSMIoT connects sensors capable of measuring environmental conditions, such as temperature, humidity to support smart farming solutions in emerging markets [4].

GSM is based on the frequency reuse mechanism, thanks to this principle that an operator can increase the capacity of its

Manuscript received February 17, 2020; revised March 17, 2020. Date of publication July 8, 2020. Date of current version July 8, 2020. The associate editor prof. Miroslav Joler has been coordinating the review of this manuscrip and approved it for publication.

H. Megnafi is with the Telecommunication Laboratory of Tlemcen (LTT), University of Tlemcen and Higher School in Applied Sciences (ESSATlemcen), Algeria (E-mail: h.megnafi@essa-tlemcen.dz).

Digital Object Identifier (DOI): 10.24138/jcomss.v16i3.1012 network [5]. This principle makes it possible to avoid saturation in the cells when the number of subscribers increases to minimize interference effects between channels [6]. It is an optimization problem that consists in choosing an optimal frequency plan in order to meet traffic demand constraints and quality of communication while minimizing the co-channel and adjacent channel interference [7], [8].

The planning tools have frequency planning algorithms used in the program proposal of frequency plan such as ATOLL. These tools are based on the integration of propagation models adapted to many environments, and taking into account the reflection phenomena [9], ground diffraction, etc. The planning tool is becoming essential for offering a high quality service network [10]. This tool requires the introduction of several parameters for its proper functioning, such as the basic network infrastructure, the digital model of a geographic area to plan [11], the digital map with morphological and topographic information, etc. In practice, the digital model of a geographic area to plan which used as an input parameter, this parameter does not represent the evolution of structures in urban areas (dimensions, spacing between structures, construction materials, etc.), and likewise integrated propagation models do not take into account all the propagation phenomena of radio waves, which gives a program result of an insufficiently optimal frequency plan.

After the implementation of the frequency plan proposed in the network, radio optimization teams check the Key Performance Indicators (KPIs) of network, and they trigger an emergency drive test operation to export the results into log files in order to optimize the frequency plan obtained by the planning tool [12]. Actually, the log files analysis allows detecting co-channel and adjacent channel interference in the same area, then correcting the frequency plan and putting it back in the sites in order to optimize it. This manual verification is a continuous process, which is repeated until the final optimization of frequency plan, but this process takes a long time, and also the Quality of Service (QoS) of network decreases during this operation.

In this paper, we propose an optimization approach based on genetic algorithm. The input parameters of this approach are based on a file, which defining the adjacent cells of each cell obtained by the Operation and Maintenance Center-Radio (OMC-R), as well as the frequencies allocated to a given area. 
This approach allows proposing an optimal frequency plan in the GSM network to minimize firstly the co-channel interferences, and also to find as low adjacent channel interference as possible. Therefore, it takes less time than the manual verification.

The rest of the paper is organized as follows: Section II presents the planning and optimization in cellular network. Section III describes the optimization approach for the frequency allocation problem based on genetic algorithms. Section IV illustrates the results obtained and their analyses. Section $\mathrm{V}$ concludes the paper and presents some directions for future research.

\section{Planning \& Optimization OF Cellular Networks}

Planning a cellular network is an important step that is often repeated several times in the network life cycle, it consists in defining its coverage and capacity [13], these are vital for an operator. Therefore, the objective of network planning is to ensure traffic capacity in urban areas and to ensure the most complete coverage possible in rural areas [14].

\section{A. Radio Planning Process}

The network planning process illustrated in the Fig. 1 is an endless cycle, is divided into five main phases, four of which are located before the network launch, and the last is located after the network launch [6]. Actually, the network planning process is a never ending cycle due to changes in the design parameters.
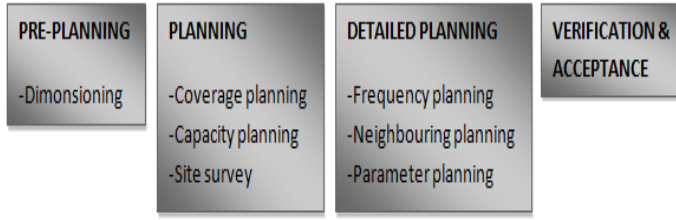
ACCEPTANCE

OPTIMIZATION
Fig. 1. Planning process steps.

The network planning process starts with the pre-planning phase, which covers assignments and preparation before the actual network planning is started. The main activity in the pre-planning phase is dimensioning, it gives a preliminary output network plan, which is then completed in the coverage and parameter planning steps to create a detailed plan [6].

The process continues with the planning phase, taking input from the dimensioning. This is the basis for nominal planning, which means radio network coverage and capacity planning with a planning tool. The nominal plan is a starting point for the site survey in order to find a real site location. The result of this phase is the final, detailed coverage and capacity plan [9]. Once the planning phase has finished and the site location is known, detailed planning can be started, which gives the frequency, neighbour and parameter plan. Therefore, the network is ready for the verification and acceptance phase, which ensures the optimal operation of network and completes the pre-launch activities. After the launch of the network, activities continue with the optimization, for this phase the needed input is all available information about the network and its status [6].

After the network has been launched, the planning and optimization related activities do not end because network optimization is a continuous process.

\section{B. Radio Optimization}

Radio optimization begins after the planning phase, is one of the main steps in improving the performance of telecommunication networks [15]. It is the determination of the rectification and solutions to performance issues, these solutions represent a change in the configuration of physical parameters (tilts and azimuths of the antennas, frequencies used in each cell, etc.), and logical parameters (selection and reselection, handover, etc.) at the Base Transceiver Station (BTS) to ensure the proper functioning of the network in terms of coverage, capacity and quality of service within the constraints of an existing network infrastructure [13].

\section{B.1 Objective of Radio Optimization}

The objective of optimization is to ensure that the network operates at optimal performance and within the defined constraints of QoS. This is achieved by implementing corrective measures and procedures to rectify identified network problems. Thus, the optimization of a cellular network is motivated by two main objectives: improving the QoS offered to users and the increase in the traffic volume carried by the network with existing equipment [16].

\section{B.2 Radio Network Optimization Process}

This process as shown in the Fig. 2 starts by monitoring network performance through statistics that are taken from the OMC-R in the GSM network, data retrieved by counters. These statistics are used to calculate its KPIs that can be defined as set of results of a formula, which is applied to Performance Indicators (PIs). The KPIs analysis allows to select the areas where there are anomalies, then locate the source of problems to develop actions to be undertaken in order to optimize the network. Sometimes, the indicators are insufficient to detect problems in the network, and then drive tests will be carried out to better understand the causes [16], although it consists of the precise characterization of radio channels. Additional drive test measurements can detect and locate the position of anomalies to provide more information about the problem. The process can also be initiated following complaints from subscribers. If the supervision system of operators does not anticipate the causes that led to these complaints, corrective actions are deduced from the KPI analysis after the channelling of anomalies.

After the validation and implementation of actions, the process starts again to see if the detected anomalies are corrected because the improvements made can cause problems 
in other parameters [13].

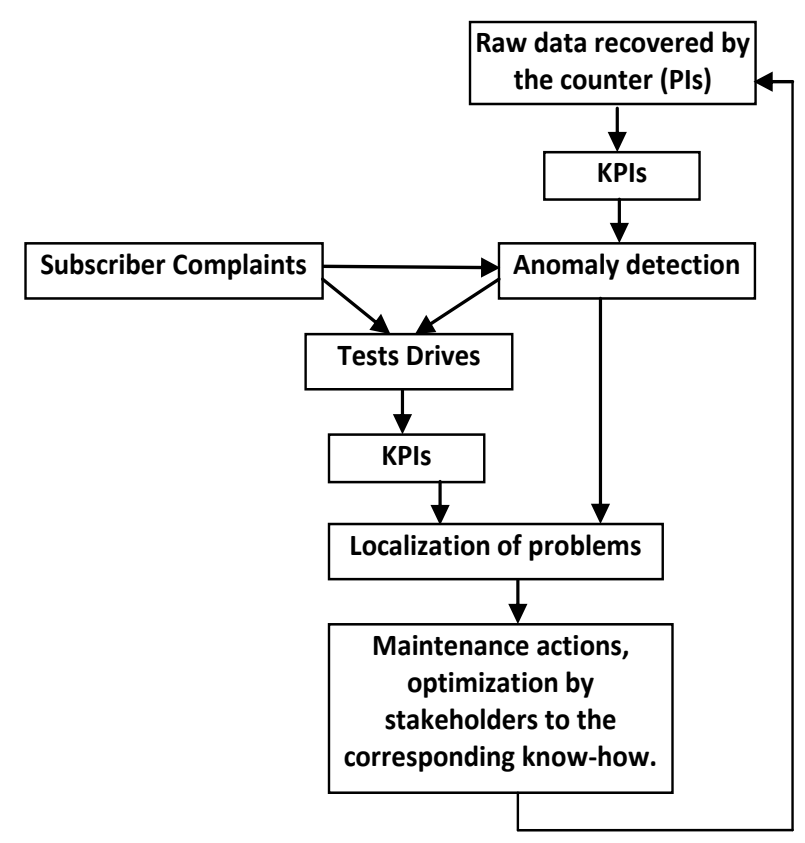

Fig. 2. General diagram of the optimization process.

\section{B.3 Drive Test}

The purpose of the drive test is to check the effectiveness of radio link in terms of coverage, quality and capacity. The drive test is run according to the pre-planned measurement route, and the tests are continued until enough data are collected for the planned analysis.

The drive test is done by a car using three specific mobiles in different modes, three external antennas placed on the roof of the car, a Global Positioning System (GPS) to localize the position of quality degradation precisely, and also a special software TEMS Investigation installed on a laptop computer for the acquisition, recording and processing of recovered measurements [17].

In the drive test operation, each mobile can be in one of three modes:

-- Idle mode: established during the drive test process, the Mobile Station (MS) is on, but no calls were made during this period.

-- Dedicated mode / long call: make continuous calls during the drive test process, and the call will not end until the end of the route.

-- Dedicated mode / short call: make short calls during the drive test process.

\section{B.4 Frequency Allocation and Reuse}

The radio resources allocation is the sensitive area of this type of system, is made by a geographical breakdown based on the hexagonal paradigm allowing frequency reuse on distant cells.

The cellular concept has made a significant contribution to network design to solve the congestion problem. In order to solve this problem, we can use identical channels (frequencies) if two cells are far enough apart to avoid generating co-channel interferences, which is called the principle of frequency reuse. Thanks to this principle, the network manages a number of simultaneous communications much larger than the number of frequencies available [14].

The principle of frequency reuse has led to several allocation strategies. There are two main techniques for managing radio resources. The first, called Fixed Channel Assignment (FCA), consists of allocating a set of nominal frequencies to each station on a permanent basis. The second, called Dynamic Channel Assignment (DCA) frequency allocation technique, no frequency assignment to stations is performed a priori [18]. Intermediate techniques have also been proposed such as Hybrid Channel Assignment (HCA) and Channel Borrowing (CB) [19].

\section{NeW APPROACH to FREQUENCY Plan OPTIMIZATION}

In order to optimize the radio part of the GSM network, we have initiated a drive test operation. After the analysis of the $\log$ files obtained by this operation, we encountered problems of co-channel and adjacent channel interference in the area due to poor frequency planning.

In this approach, we propose a technique based on the efficient use of frequency reuse, which gives an optimal frequency plan in such a way as to minimize interferences. To do this, it is necessary to define the adjacent cells before allocating the frequencies for the GSM network. In reality, adjacent cells are not only those located next to the considered cell, but there are other cells due to the reflection phenomenon of radio waves and the over-shooting problem.

In the idle mode and dedicated mode, if there is not a relationship between the new cell and the serving cell, the MS will not make a cell reselection [20]. These relationships are defined by a table configured in the BTS called neighbouring cells table. The update of this table is done by analysing the $\log$ files to verify the relationship between the serving cell and neighbouring cells in the BCCH Allocation List (BA-List) of MS, such as BCCH is Broadcast Control Channel. After selecting the table that represents adjacent cells, we can assign frequencies for each cell and propose an optimal frequency plan, but this assignment is a difficult problem to achieve [11].

There are several optimization algorithms used to obtain an approached solution to a problem [10]. In this work, we used the genetic algorithm, which consists in solving this type of problem. As the frequency planning of $\mathrm{BCCH}$ has a major impact on the radio link, the approach has been applied just to the $\mathrm{BCCH}$ frequency planning, and concerning the Traffic Channel $(\mathrm{TCH})$, we can directly activate the Frequency Hopping (FH) [18].

We present the genetic algorithm implemented in the $\mathrm{BCCH}$ frequency allocation approach for the GSM network. Initially, we create a set of randomly generated solutions called population $P$ of size $M$ (in our case $M=40$ ), and each solution is represented by a chromosome of size $\mathrm{N}$, such that $\mathrm{N}$ is the number of cells to plan. A chromosome is made up of a set of genes, the numeric value of genes represents the Absolute Radio Frequency Channel Number (ARFCN) BCCH of a 
given cell, which is between 1 to 124 for GSM900 and 512 to 885 for GSM1800 [21]. The first gene of chromosome represents the $\mathrm{BCCH}$ frequency (or $\mathrm{ARFCN} \mathrm{BCCH}$ ) of the first cell, and the second gene represents the $\mathrm{BCCH}$ frequency of the second cell, etc. The Fig. 3 shows an example of a chromosome, for this solution the $\mathrm{BCCH}$ frequency for the first cell is equal to 80 , and for the second cell is equal to 82 , etc.

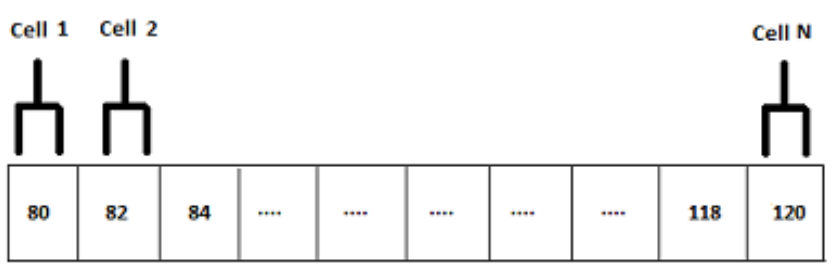

Fig. 3. A chromosome example.

The Evaluation of all solutions for population $\mathrm{P}$ is summed up to one criterion because of the frequency range choice dedicated to the $\mathrm{BCCH}$ channels. In practice, the radio optimization teams choose even frequencies for $\mathrm{BCCH}$ and odd frequencies for $\mathrm{TCH}$, this choice is made to avoid the problem of adjacent $\mathrm{BCCH}$ (the activation of $\mathrm{FH}$ allows to solve the problem of adjacent frequencies between the $\mathrm{BCCH}$ and $\mathrm{TCH}$ ).

The fitness function (also known as the evaluation function) is calculated by adding the numbers of co-channel $\mathrm{BCCH}$ present in each gene of chromosome. This calculation is based on the $\mathrm{BCCH}$ frequencies assigned to each gene (which presents a cell) and neighbouring cells table. It is given by the following function:

$F=\sum_{i=1}^{N} \sum_{j=i+1}^{N} T(i, j) K$

Where $\mathrm{F}$ represents the fitness function value of the cochannel $\mathrm{BCCH}$ for chromosome, $\mathrm{N}$ represents the size of chromosome. $\mathrm{T}(\mathrm{i}, \mathrm{j})$ is a square matrix of $\mathrm{N}$ dimensions obtained by the neighbouring cells table, it represents the neighbour cells for each serving cell and $\mathrm{K}$ represents the equality of the genes values between cell $\mathrm{i}$ and cell $\mathrm{j}$ :

$T(\mathrm{i}, \mathrm{j})= \begin{cases}1 & \text { if cell } \mathrm{i} \text { is adjacent to cell } \mathrm{j} \\ 0 & \text { if cell } \mathrm{i} \text { is not adjacent to cell } \mathrm{j} \text { or } \mathrm{i}=\mathrm{j}\end{cases}$

$K= \begin{cases}1 & \text { if chromosome }(\mathrm{i})=\text { chromosome }(\mathrm{j}) \\ 0 & \text { if chromosome }(\mathrm{i}) \neq \text { chromosome }(\mathrm{j})\end{cases}$

A mutation operation acts by randomly modifying one or more genes on a chromosome, while the crossover swaps some genes from one parent with those from another. Each pair of individuals in the population of selection is associated with a probability of performing the crossover $P_{c}$ (its value is 0.5 ), and the individuals generated constitute the population $\mathrm{G}$, after that, each gene in an individual of $G$ is mutated before being inserted into the population with a probability $\mathrm{P}_{\mathrm{m}}$ (its value is 0.01) [15]. The individuals $G$ then represent the new population and the cycle continues: evaluation, selection, crossover, mutation, etc.

After several generations, the chromosome value will converge to a certain value, which is the best solution [14].

The different steps of the genetic algorithm process are described in the Table I.

TABLE I

GENETIC ALGORITHM PROCESS

\begin{tabular}{ll}
\hline \hline Steps & Operation \\
\hline Step 1 & Generate the initial population $\mathrm{P}$ of size $\mathrm{M}$ randomly. \\
Step 2 & Evaluate all solutions in population $\mathrm{P}$.
\end{tabular}

Select both parents using the "binary selection per tournament"

Step 3 It consists in randomly drawing two solutions from the population $\mathrm{P}$ then selects the solution with the highest rank.

Step 4 Generate two solutions of children by crossing the two parent solutions with a probability $\left(\mathrm{P}_{\mathrm{c}}\right)$.

Step 5 Execute the transfer operator with a probability for each child solution $\left(\mathrm{P}_{\mathrm{m}}\right)$.

Step 6 Add the two child solutions to the following population G.

Step 7 Repeat steps 3 to 6 to obtain $\mathrm{N}$ child solutions in population G

Step 8 Repeat steps 2 to 7 until the number of iterations is reached.

\section{RESUlts OBTAINED \& ANALYSES}

To validate the proposed approach, a drive test operation has been triggered to evaluate the level of the received signal level (Rxlevel) and the Carrier to Interference worst (C/I worst) in an area.

The drive test was carried out for 8 sites, which are located in the same area with three mobiles in different modes (short calls, long calls in dedicated mode and idle mode) in order to study the different distributions of measured radio parameters.

The drive test requires testing the network in a particular area to provide a true picture of the network. The drive test operation gives us the distribution of the different parameters in the map, which represents the target territory such as RxLevel, C/I worst. To facilitate the analysis of the drive test, we have separated the values of each parameter into levels, and each of these is associated by a colour. The Fig. 4 shows the trace route of the drive test operation.

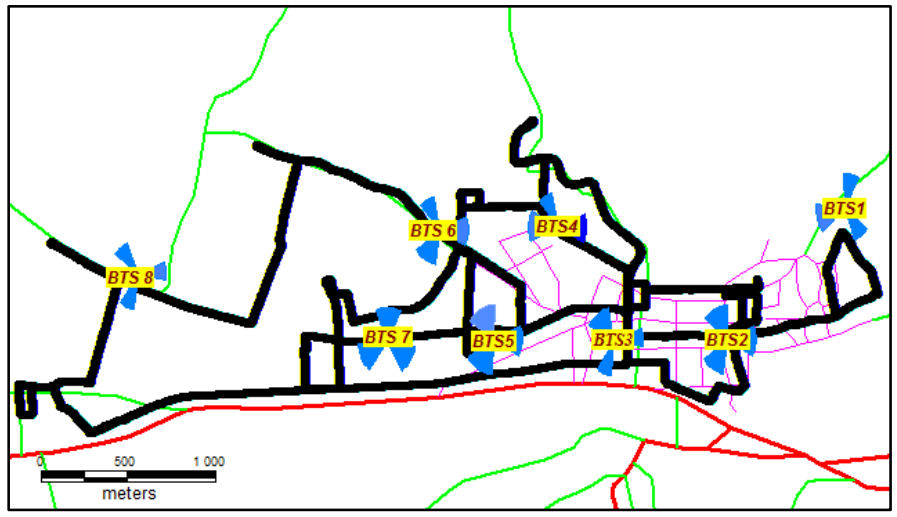

Fig. 4. Trace route of the considered area. 
The choice of trace route for a given region was taken into account all main landforms, the different heights of the landforms and the different positions of sites.

The Fig. 5 shows the distribution of RxLevel in $\mathrm{dBm}$ in dedicated mode / long call on the route.

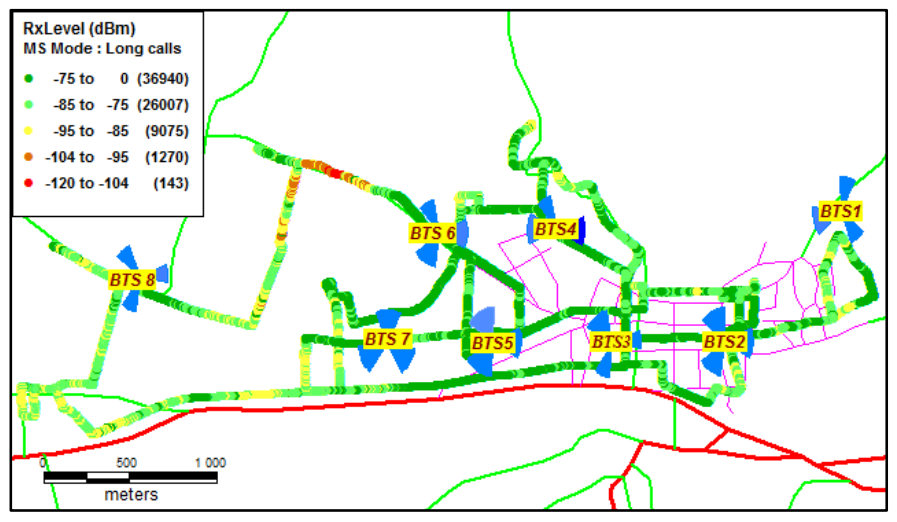

Fig. 5. Received signal level in long call mode on the route.

We have classified the signal level into five ranges as shown in the legend of Fig. 5. The number of recorded samples characterized by a signal level below $-85 \mathrm{dBm}$ is negligible compared to the one that is greater than $-85 \mathrm{dBm}$, the number shows that the signal level distribution is better.

The Fig. 6 shows the distribution of C/I worst in dedicated mode / long call on the route.

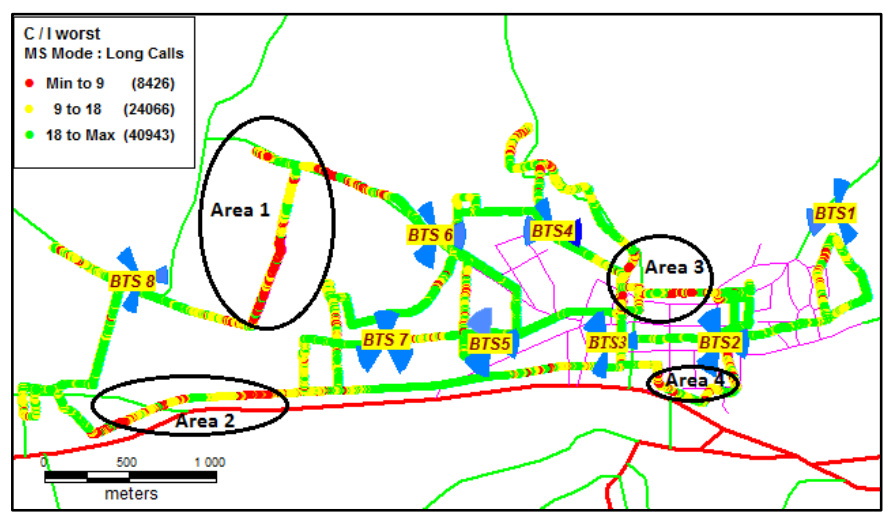

Fig. 6. C/I worst in long call mode on the route.

The Fig. 6 above shows the distribution of the term C/I worst in a region. We notice a presence of red sections indicating a low term $\mathrm{C} / \mathrm{I}$ worst from less than $9 \mathrm{~dB}$ (area 1, 2, 3 and 4), which means that there is a radio interferences caused by a poor coverage, or a frequency planning problem in this region. However, the distribution of the received signal RxLevel in the Fig. 5 does not represent a coverage problem (area 1, 2, 3 and 4), it therefore means that the problem is due to a poor frequency plan.

In order to validate the cause of the problem in this region, we analysed the log files through TEMS Investigation software, we clearly see that there is a co-channel interference between cell C of BTS 1 and cell A of BTS 6 , and similarly between cell B of BTS 4 and cell A of BTS 8 as shown in the
Fig. 7.

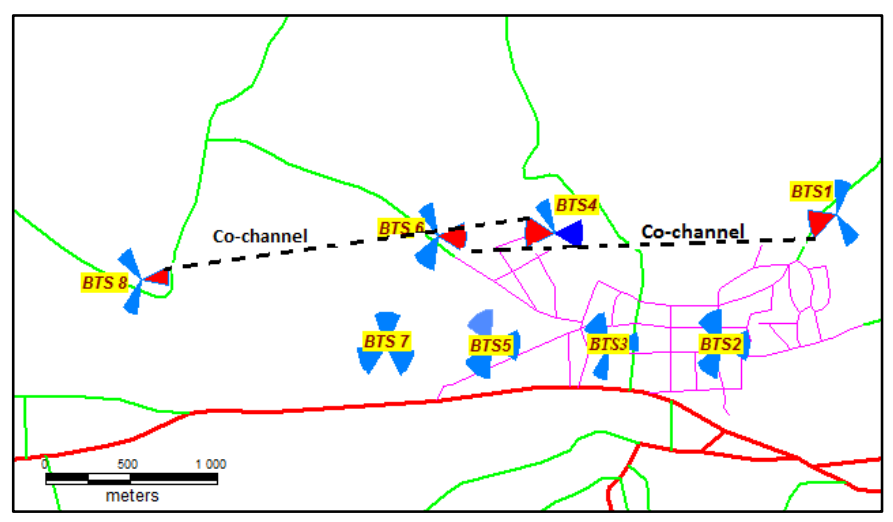

Fig. 7. Presentation of co-channel interferences in the considered area.

In order to solve the co-channel interference problem in this area, we have proposed an optimization approach based on genetic algorithms, which is implemented in $\mathrm{C}++$ language. This approach allows proposing an optimal frequency plan based on two input parameters. The first is the initial frequency plan of $\mathrm{BCCH}$ frequencies implemented in this area, and the second is the neighbouring cells table.

The network is composed of a set of 8 BTS, for a total of 24 cells, whose telephone operator has 15 frequencies dedicated to the $\mathrm{BCCH}$ channel and 22 frequencies on the TCH channel in this area. The neighbouring cells table is given in the Table II.

TABLE II

The NeIGHBOURING CELls TABLE.

\begin{tabular}{cl}
\hline \hline Cell & Neighbouring cells \\
\hline 1 & $2,3,10,4,6,7,16,13,22,19$ \\
2 & $1,3,4,5,6,10,7,13,16$ \\
3 & $1,2,10,7,6,16,13,19,22,16$ \\
4 & $5,6,2,10,7,16,13,1$ \\
5 & $4,6,7,8,14,20,16,19,2,11,3$ \\
6 & $3,7,10,11,13,16,22,19$ \\
7 & $8,9,3,10,6,16,22,2$ \\
8 & $7,9,5,14,11,16,19,7$ \\
9 & $7,8,13,11,16,22,19,3,12$ \\
10 & $2,3,6,4,7,9,11,12,16,22$ \\
11 & $10,12,3,6,9,13,19,17,16,22,21$ \\
12 & $9,6,16,24,18,15,21,10,11$ \\
13 & $16,19,3,9,11,10,6,14,15$ \\
14 & $13,15,19,20,8,5,11,17,23$ \\
15 & $13,14,19,21,17,23,11,9,3,8$ \\
16 & $15,13,21,17,18,11,3,9,7,15,6,3$ \\
17 & $16,18,11,9,21,15,23,19,14,13$ \\
18 & $9,12,6,12,24,3,16,17$ \\
19 & $13,14,19,21,17,23,11,9,3,8$ \\
20 & $20,21,14,15,13,9,8,17,11,3,5,6$ \\
21 & $19,20,23,17,15,11,9,22,24,18,16$ \\
22 & $23,24,17,18,16,12,11,21,15,3,9$ \\
23 & $22,24,17,21,20,15,9,11,8,3,5,14,5$ \\
24 & $22,23,18,12,3,6,9,15,21,17,20$ \\
\hline
\end{tabular}

The table is made up of two columns, the first of which represents the serving cells, the second represents all adjacent 
cells of each cell. For example, the cell 2 (sector B of BTS 1) has a relationship with cell 1 (sector A of BTS 1), cell 3 (sector C of BTS 1) and cell 4 (sector A of BTS 2), etc.

The update of this table is done by the analysis of log files obtained by the drive test operation, which means exactly to check the cells of BA-List that are in better situations (high signal level), and which have not been selected to be a serving cells.

After loading the initial frequency plan and the neighbouring cells table in the application, we noticed in the genetic algorithm angle that there is a convergence of results (zero cochannel interference) from chromosome 37 till the chromosome 40. The final solution is represented in the frequency plan result angle. The Fig. 8 shows an execution capture of the application in GSM network.

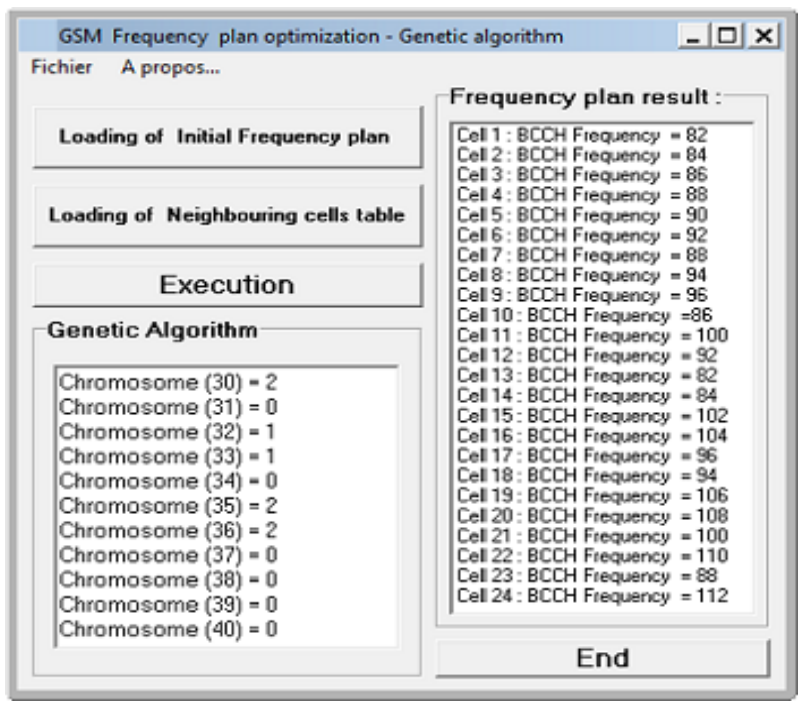

Fig. 8. Capture of the implemented application.

With applying the frequency plan obtained by the genetic algorithm in the network, a drive test operation was triggered in order to check the term $\mathrm{C} / \mathrm{I}$ worst in the area. The Fig. 9 shows the distribution of $\mathrm{C} / \mathrm{I}$ worst.

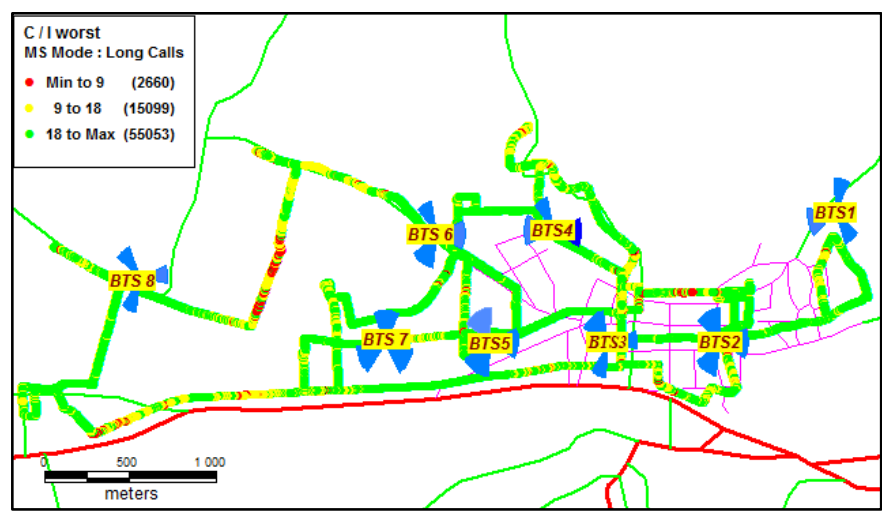

Fig. 9. C/I worst in long call mode on the route.

We notice in the Fig. 9 that the number of samples recorded less than $9 \mathrm{~dB}$ is negligible compared to the number of samples recorded greater than $18 \mathrm{~dB}$, and therefore we obtained no interference (zero co-channel interference), and we have also succeeded in improving the term C/I worst.

\section{CONCLUSION}

In this study, it was demonstrated that planning software based on environmental modelling and radio wave propagation models do not give an optimal frequency plan. In addition, there is a need for an effective approach, which minimizes co-channel and adjacent channel interference in the network in order to optimize the frequency plan. In this regard, the proposed optimization method is based on genetic algorithms with the use of the BA-List table, which allows to actually define the neighbouring cells for each serving cell in the network. The validation of the approach has been confirmed by the work applied in GSM network of MOBILISAlgeria operator to solve an optimization problem. The results obtained are encouraging because this developed approach finds better solutions.

As future scopes for this work, other frequency allocation approaches based on different heuristics (tabu search metaheuristic) can be developed. It is also possible to use in this approach the export of log files obtained by the drive test operation to better optimize the frequency plan.

\section{REFERENCES}

[1] Rose, M. Dennis, S. Hahn, and T. Kürner: "Evolution from network planning to SON management using the simulator for mobile networks (SiMoNe) ", 2016 IEEE 27th Annual International Symposium on Personal, Indoor, and Mobile Radio Communications (PIMRC) IEEE, pp. 1-2, 2016, doi: 10.1109/PIMRC.2016.7794575

[2] S. Hämäläinen, H. Sanneck, and C. Sartori: "LTE self-organising networks (SON): Network management automation for operational efficiency", Wiley, 2012.

[3] Petrenko, S. Alexey, Petrenko, A. Sergei, Makoveichuk, and A. Krystina: "The IIoT/IoT device control model based on narrow-band IoT (NB-IoT)", 2018 IEEE Conference of Russian Young Researchers in Electrical and Electronic Engineering (EIConRus), IEEE, pp. 950953, 2018, doi : 10.1109/EIConRus.2018.8317246

[4] W. Ayoub, A. Samhat, F. Nouvel, M. Mroue, and J.-C. Prévotet: "Internet of mobile things: Overview of lorawan, dash7, and nb-iot in lpwans standards and supported mobility", IEEE Communications Surveys \& Tutorials, Vol.21, No.2, pp.1561-1581, 2018, doi: 10.1109/COMST.2018.2877382

[5] Jorswieck, A. Eduard, L. Badia, T. Fahldieck, E. Karipidis, and J. Luo: "Spectrum sharing improves the network efficiency for cellular operators", IEEE Communications Magazine, Vol.52, No.3, pp.129136, 2014, doi: 10.1109/MCOM.2014.6766097

[6] H. Zhang, Y. Qiu, Y. Chu, K. Long, and V. C. Leung: "Fog radio access networks: Mobility management, interference mitigation, and resource optimization", IEEE Wireless Communications, Vol. 24, No.6, pp. 120127, 2017, doi: 10.1109/MWC.2017.1700007

[7] P. Ghamisi, and J.A. Benediktsson: "Feature selection based on hybridization of genetic algorithm and particle swarm optimization", IEEE Geoscience and remote sensing letters, Vol.12, No.2, pp.309-313, 2014, doi: 10.1109/LGRS.2014.2337320

[8] Y. Yoon and Y.-H. Kim: "An efficient genetic algorithm for maximum coverage deployment in wireless sensor networks", IEEE Transactions on Cybernetics, Vol.43, No.5, pp.1473-1483, 2013, doi: 10.1109/TCYB.2013.2250955

[9] Mohammed, M. Elbagir, and K.H. Bilal: "LTE Radio Planning Using Atoll Radio Planning and Optimization Software", International Journal of Science and Research (IJSR), Vol. 3, No. 10, pp. 1460-146, 2014.

[10] A. Esposito, L. Tarricone, and M. Zappatore: "Software Agents: Introduction and Application to Optimum 3G Network Planning", IEEE Antennas and Propagation Magazine, Vol. 51, No. 4, pp. 147-155, 2009, doi: 10.1109/MAP.2009.5338708 
[11] Mishra, R. Ajay: "Advanced cellular network planning and optimisation: 2G/2.5 G/3G... evolution to 4G", John Wiley \& Sons, 2007.

[12] Popoola, I. Segun, Atayero, A. Aderemi, N. Faruk, and J.A. Badejo: "Data on the key performance indicators for quality of service of GSM networks in Nigeria", Data in brief, Vol. 16, pp. 914-928, 2018, doi: 10.1016/j.dib.2017.12.005

[13] I. Siomina and D. Yuan: "Analysis of Cell Load Coupling for LTE Network Planning and Optimization", IEEE Transactions on Wireless Communications, Vol. 11, No. 6, pp. 2287-2297, June 2012, doi: 10.1109/TWC.2012.051512.111532.

[14] I. Amro and W. Ghanem: "GSM Automatic Frequency Planning with the Aid of Genetic Algorithms", 2011 Fifth International Conference on Genetic and Evolutionary Computing, Xiamen, 2011, pp. 73-76, doi: 10.1109/ICGEC.2011.26.

[15] F. Luna, Nebro, J. Antonio, E. Alba, and J. Durillo: "Solving largescale real-world telecommunication problems using a grid-based genetic algorithm", Engineering Optimization, Vol.40, No.11, pp.1067-1084, 2008, doi: 10.1080/03052150802294581

[16] Mishra, and R. Ajay: "Cellular technologies for emerging markets: $2 \mathrm{G}$ 3G and beyond", John Wiley \& Sons, 2010.

[17] C. Skianis: "Introducing automated procedures in $3 \mathrm{G}$ network planning and optimization", Journal of Systems and Software, Vol. 86, No. 6, pp. 1596-1602, 2013, doi: 10.1016/j.jss.2013.01.072

[18] U. Mehboob, J. Qadir, S. Ali, and A. Vasilakos: "Genetic algorithms in wireless networking: techniques, applications, and issues", Soft Computing, Vol.20, No.6, pp.2467-2501, 2016, doi: 10.1007/s00500016-2070-9

[19] L. Asadzadeh: "A local search genetic algorithm for the job shop scheduling problem with intelligent agents", Computers \& Industrial Engineering, Vol.85, pp.376-383, 2015, doi: 10.1016/j.cie.2015.04.006

[20] Huang, Han-xiong, J.C. Li, Xiao, and Cheng-Long: "A proposed iteration optimization approach integrating backpropagation neural network with genetic algorithm", Expert Systems with Applications, Vol. 42, No. 1, pp. 146-155, 2015, doi: 10.1016/j.eswa.2014.07.039

[21] F. Garzia, C. Perna, and R. Cusani: "Optimization of UMTS network planning using genetic algorithms", Communications and Network, Vol.2, No.03, pp.193, 2010, doi: 10.4236/cn.2010.23028

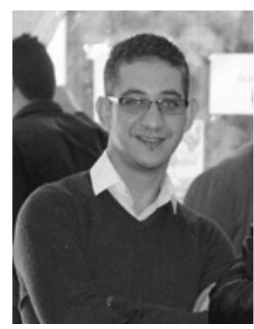

Hicham Megnafi (Ph.D. in Telecommunications) is a Lecturer-researcher at the higher school in applied sciences in Tlemcen - Algeria and member of the Tlemcen Telecommunications Laboratory (LTT). He is working in the field of $2 \mathrm{G} \& 3 \mathrm{G}$ networks and having more than 7 years of experience. He worked as radio network optimization engineer in MOBILIS Operator ALGERIA and having 4 years of experience. He worked also on Radio network optimization Consulting with ERICSSON and having 3 years of experience. His research interests include software engineering wireless, cellular communications, radio network optimization, Internet of Things and UAVs development for telecommunication applications. 